\title{
Identification of Turning and Milling Processes by Stochastic Langevin Equations
}

\author{
Grzegorz Litak and Rafał Rusinek \\ Department of Applied Mechanics, \\ Lublin University of Technology, PL-20-618 Lublin, Poland \\ Email: g.litak@ pollub.pl, http://litak.pollub.pl
}

\begin{abstract}
We analyze experimental results of cutting and milling processes. To estimate the region of chatter appearance we perform analysis of corresponding signals using the suitable stochastic Langevin equations. This procedure, in which a nonparametric model is considered, provides tools for estimating a machining process noise level. It was shown and noise level and instabilities in the process of turning and/or milling are important aspects of the formation of chatter vibration.
\end{abstract}

\section{INTRODUCTION AND EXPERIMENTAL STANDS}

The aim of this study is to estimate the level of noise arising in the process of turning and milling [1] based on the identification of deterministic and random components of the Langevin equation $[2,3]$.

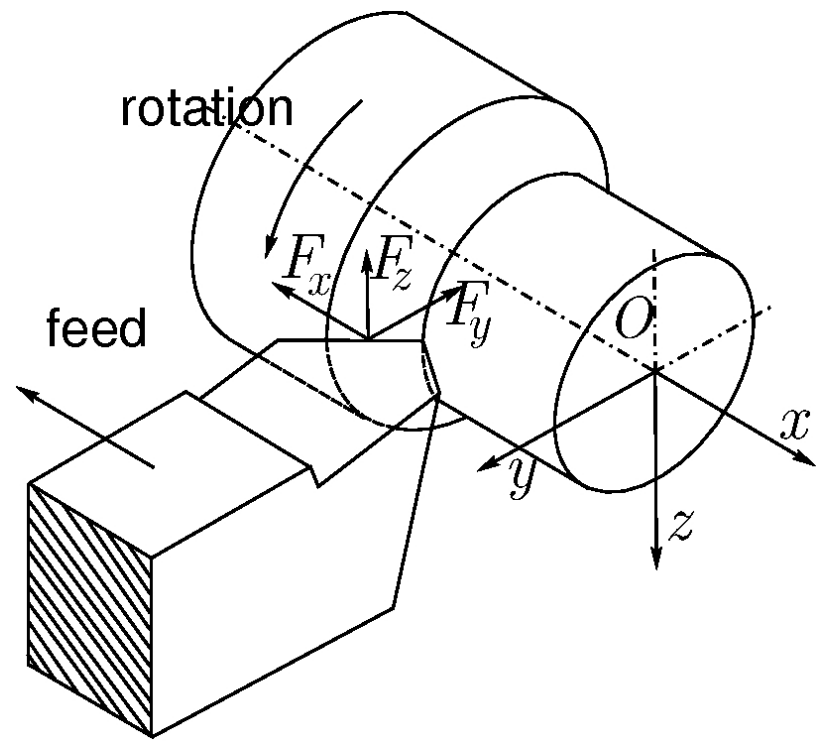

Fig. 1. Schematic picture of the model used in the turning process The directions used in the process description and the cutting force components are also presented [4].

The turning experiment (Fig. 1) was carried out on a round stainless steel shaft (EZ6NCT25) with a diameter of $22 \mathrm{~mm}$. The tool tip at an angle of 45 degrees. Angular velocity of the shaft was set at about 780 rev per minute (rpm), while the corresponding feed rate was $0.25 \mathrm{~mm} / \mathrm{rev}$. The experiment was repeated for three depths of cut $h_{0}=1.00,1.75$, and 2.30 $\mathrm{mm}$.

The results of measurements of the radial component of cutting force $F_{y}$ in the process of turning [4] with varying

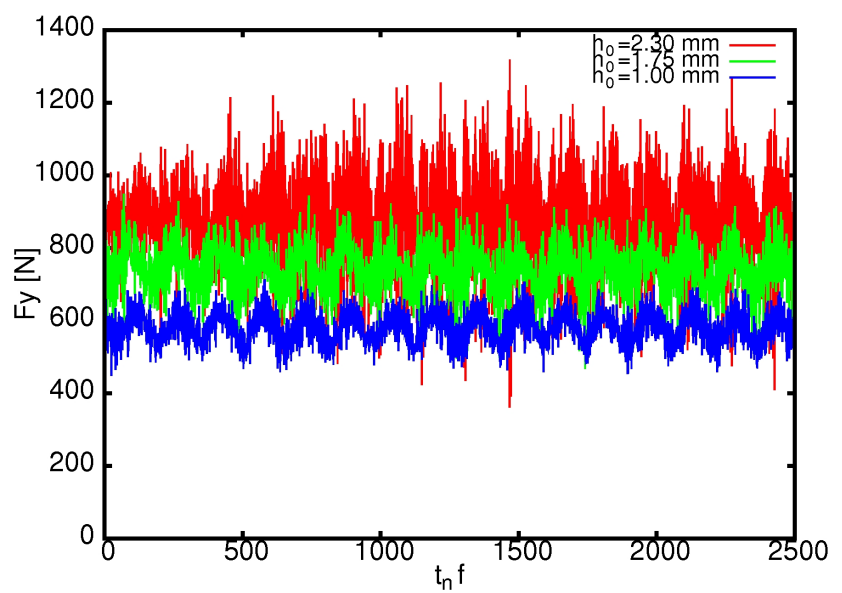

Fig. 2. Time series of the radial component of a cutting force $F_{y}$ for different cutting depths $h_{0}\left(h_{0}=1.00,1.75\right.$, and 2.30 -plotted in blue, green, and red colours, respectively). $n$ is the number of subsequent force measurement, measuring discrete time $t_{n}=n / f$, where the sampling frequency was $f=2 \mathrm{kHz}$.

cutting depth $h_{0}\left(h_{0}=1.00,1.75\right.$, and $\left.2.30 \mathrm{~mm}\right)$ are presented in Fig. 2 It is worth noting that with increasing $h_{0}$ increases also the average force $\left\langle F_{y}\right\rangle\left(<F_{y}\right\rangle=581.2,740.9$, and $868.6 \mathrm{~N})$ as well as the amplitude of the fluctuations measured by the value of standard deviation $\left(\sigma_{y}=45.0,68.8\right.$, and $151.1 \mathrm{~N})$.

Other experiments on a milling process (Fig. 3) were performed in the system consisting of a CNC machine, a piezoelectric dynamometer to measure strength, charge amplifier, data collection module, and a standard digital converter and the computer to record the results. Milled samples were based on of the epoxy polymer matrix composite reinforced with carbon fibers (EPMC). Measurements were performed for different angular speeds, $\omega=2000,3500$ and 8000rpm at a constant feed of $520 \mathrm{~mm} / \mathrm{min}$ [5]. The relevant series of cutting force component $F_{x}$ is shown in Fig. 4a-c. Note that the force component $F_{x}$ takes both positive and negative values. The corresponding average values are rising $\left\langle F_{x}\right\rangle$ $=6.49,11.56$ and $12.05 \mathrm{~N}$, and the standard deviations show a non-monotonic behavior $\sigma_{x}=22.67,24.88$ and $21.99 \mathrm{~N}$.

Figure 4 shows the modulated vibration. Longer period oscillations mirror time scale strictly connected to the total 


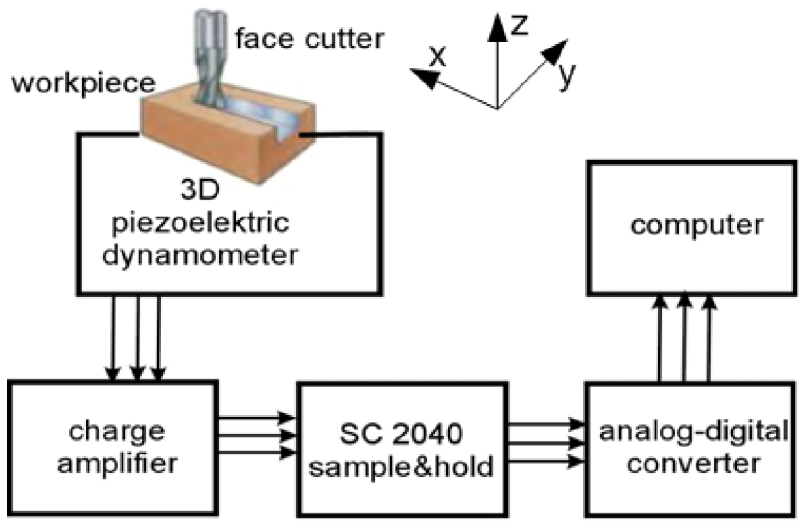

Fig. 3. The diagram of the measurement procedure in the milling process with a corresponding coordinate system.
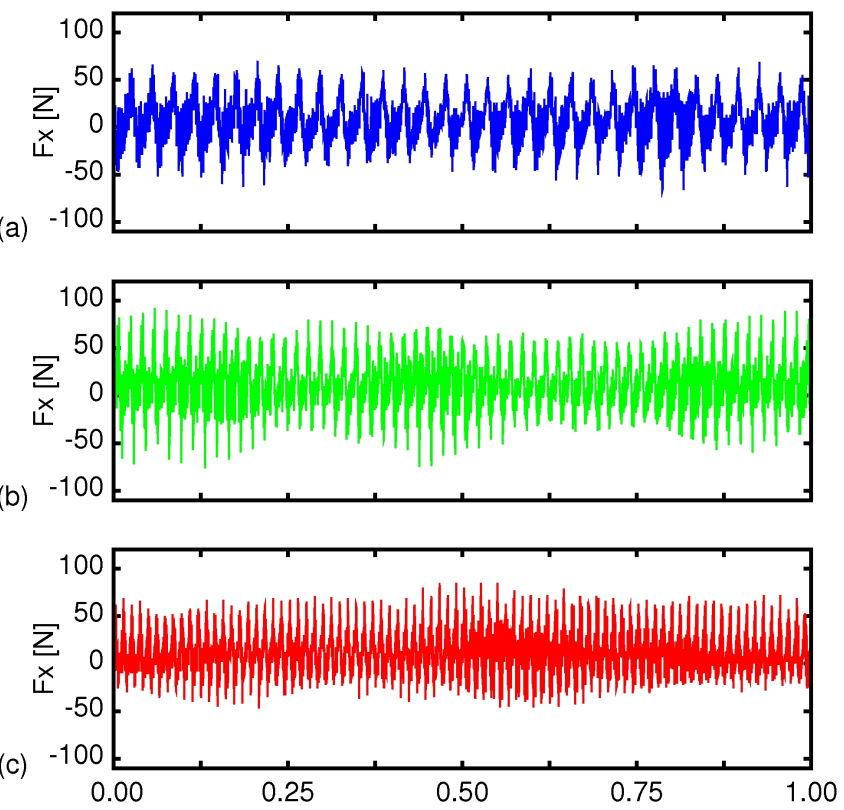

Fig. 4. Cutting force component $F_{x}$ for speeds $\omega=2000,3500$, $5000 \mathrm{rpm}$ are plotted in (a)-(c) respectively. Sampling frequency was $f=4 \mathrm{kHz}$.

turnover of a cutter due to a regenerative time delay. Short time changes include the relative displacement between tool and workpiece which could have deterministic or stochastic character. By increasing $\omega$ (Fig. 4a to 4c) we expect more rotations (with fixed sampling rate). Similar features but of different time scale can be seen also in the treatment of turning (Fig. 2).

\section{Application of A Stochastic LANGEvin EQuation}

For a large class of random processes, it is possible to describe the stochastic differential equation of the first order $[2,3]$

$$
\frac{\mathrm{d}}{\mathrm{d} t} \mathbf{X}=\mathbf{h}(\mathbf{X}(t))+\mathbf{g}(\mathbf{X}(t)) \mathbf{\Gamma}(t)
$$

where $X(t)$ denotes the $D$-dimensional randomly changing system coordinate, and $\mathbf{h}($.$) and \mathbf{g}(.) \boldsymbol{\Gamma}(t)$ indicate the drift and diffusion terms. In particular, $\mathbf{g}($.$) denotes the correlation$ matrix, while $\Gamma(\mathrm{t})$ symbolizes a stochastic process with Gauss distribution.

An interesting feature of Fig. 1 is that it can be used in both ways, to simulate and identify solutions or to characterize experimental measurements. In the following analysis we apply this equation to identify the level of noise in cutting (Fig. 2) and milling (Fig. 4) processes. In our analysis the Langevin equation will identify values of $\mathbf{h}($.$) and \mathbf{g}($. terms for the corresponding time series. We assume that the appropriate components of cutting forces $F_{i}$ correspond to the representation of $X(t)$ (scalar representation of the vector $\mathbf{X}(t)$ ).

For the present analysis we assume the process of turning the experimental results obtained in the respective measurement times $t_{n}=n \Delta t$, where $n$ is the current number measured force and $\Delta t$ is the time interval between successive measurements. Therefore, the coordinate describing the change of state (Eq. 1) can be expresses as follows [2]:

$$
X\left(t_{n}\right)=F_{y}(n)
$$

Then

$$
\begin{gathered}
g\left(\phi_{n^{\prime}}\right)=<\left(X\left(t_{n+1}\right)-X\left(t_{n}\right)\right)^{2}>, \\
h\left(\phi_{n^{\prime}}\right)=<\left(X\left(t_{n+1}\right)-X\left(t_{n}\right)\right)>,
\end{gathered}
$$

where the averaging $\langle$.$\rangle is carried out for the appropriate$ angle $\phi_{n^{\prime}}$ associated with the fairly large number of measurements $N$ on the sample rotation or turning of the milling cutter to machine (Fig. 5) the number of cycles.

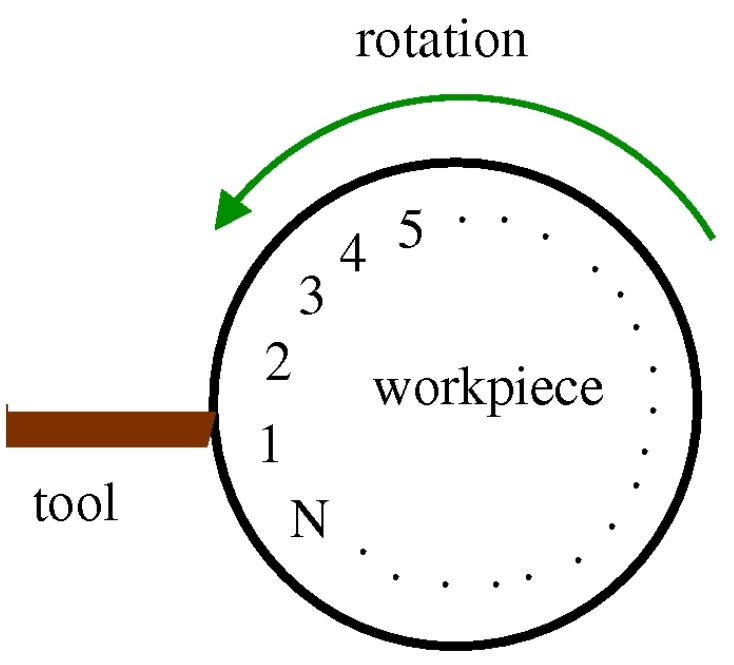

Fig. 5. Schematic diagram of an exemplary selection of discrete angle measurement ( $n^{\prime}$ ranges from 1 to $N_{0}$ corresponding to the choice of a particular angle $\phi_{n^{\prime}}=n^{\prime} \phi_{0}$, where $\phi_{0}$ is elementary angle.) for averaging.

Using the Eq. (3) to the corresponding waveforms (time series) in Fig. 2 and 4 were obtained characteristic diffusion 
coefficients $g\left(n^{\prime}\right)=g\left(\phi_{n^{\prime}}\right)$. In the case of the rolling process can be seen a marked increase $g$ for increasing cutting depth (Fig. 6). The average value $\left\langle g\left(n^{\prime}\right)>=62.7,104.5\right.$ and 243.9 $\mathrm{N}$ is doubled at the transition to the next value of $h_{0}$. This results show the monotonous increase of noise-to-signal value $<g>/<F_{y}>$.

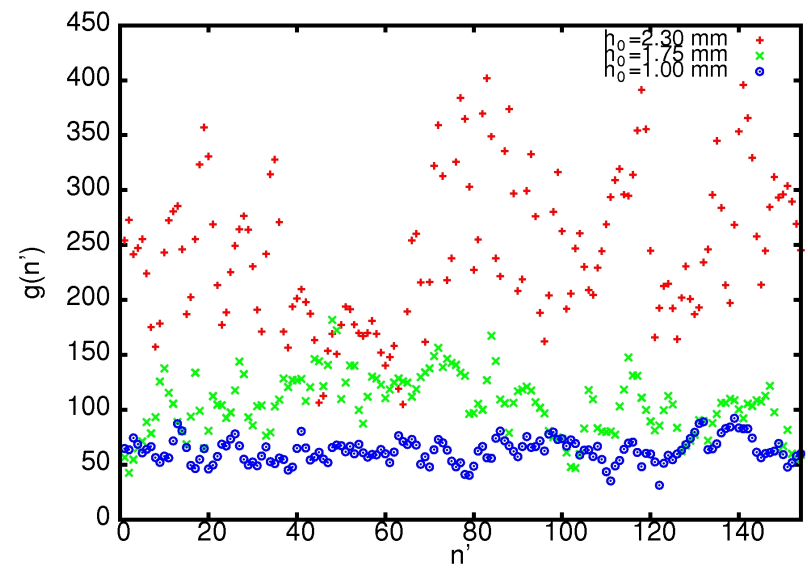

Fig. 6. The dependence of noise levels $g\left(n^{\prime}\right)$ of the angle measurement $n^{\prime} \in\left[1, N_{0}\right]$ for turning the results corresponding to the time series shown in Fig. 2 for different cutting depth $h_{0}$. Here $N_{0}=154$ means that 154 measurements were correspond to a single revolution (Fig. 5). $g\left(n^{\prime}\right)$ is expressed in units of $[\mathrm{N}]$.

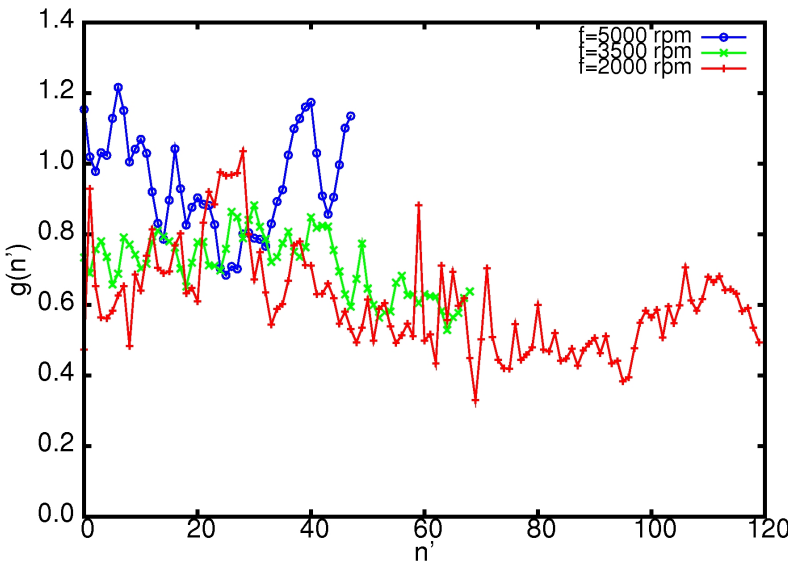

Fig. 7. The dependence of noise levels $g\left(n^{\prime}\right)$ of the angle measurement $n^{\prime} \in\left[1, N_{0}\right]$ for turning the results correspond to the ranks of the time shown in Figure 4 for different angular speeds $\omega=$ 2000, 3500 and 5000. Consequently, number of measurement events per revolution $N_{0}$ takes the following values 48,69 and 120 in one revolution (Fig. 5). $\mathbf{g}\left(n^{\prime}\right)$ is expressed in units of $[\mathrm{N}]$.

The examined milling process is also an interesting case. Here the noise increases with increasing speed $\omega=2000$, $3500,5000 \mathrm{rpm}$. The corresponding average $\left\langle g\left(n^{\prime}\right)\right\rangle=0.61$, $0.72,0.94 \mathrm{~N}$. Thus, the noise level definitely goes up. However on the basis of the standard deviation of force $F_{x}$, noise-to- signal value $\langle g\rangle /\left\langle F_{x}>\right.$ riches minimum at the rotation speed $\omega=350$.

\section{Summary AND CONCLUSIONS}

Basing on the Langevin equation, we performed the measured signal analysis. Note that the equation, consisting with the drift and diffusion terms, was used as a non-parametric model. It provided an information about the noise level and simultaneously about the system stability in a machining process. This paper presents the results of this analysis for turning and milling processes. It is worth noting that the noise and instability in the process of turning and milling process are important aspects in forming of chatter vibration [1]. In particular, the heterogeneous and hardly machinable materials, which include composites and stainless steel were analyzed. For these materials are observed higher periodic and stochastic components of oscillations $[4,5]$. To apply the above procedure in practice we need to perform more systematic studies taking into account all components of the forces.

\section{ACKNOWLEDGMENT}

The financial support of Structural Funds in the Operational ProgrammeInnovative Economy (IE OP) financed from the European Regional Development Fund Project Modern material technologies in aerospace industry, No. POIG.01.01.0200-015/08-00 is gratefully acknowledged.

\section{REFERENCES}

[1] Y. Altintas Y, Manufacturing Automation: Metal Cutting Mechanics, Machine Tool Vibrations, and CNC Design, Cambridge University Press, Cambridge 2000.

[2] J. Gradisek, R. Friedrich, E. Govekar, and I. Grabec, "Analysis of data from periodically forced stochastic processes", Physics Letters A 294, pp. 234-238, 2002.

[3] J. Lipski, G. Litak, R. Rusinek, K. Szabelski, A. Teter, J. Warmiski, and K. Zaleski, "Surface quality of a work materials influence on vibrations in a cutting process", Journal of Sound and Vibration 252, pp. 729-737, 2002.

[4] G. Litak and R. Rusinek R. "Dynamics of a stainless steel turning process by statistical and recurrence analyses", Mechanica 47, pp. 1517-1526, 2012.

[5] G. Litak, A. Syta, and R. Rusinek, "Dynamical changes during composite milling: Recurrence and multiscale entropy analysis", International Journal of Advanced Manufacturing Technology 56, pp 445-453, 2011. 
G. Litak and R. Rusinek • Identification of Turning and Milling Processes by Stochastic Langevin Equations 